UCRL-JC-130031

Preprint

\title{
The National Ignition Facility (NIF) Wavefront Control System
}

R. Zacharias, E. Bliss, M. Feldman, A. Grey, M. Henesian, J. Koch, J. Lawson, R. Sacks, T. Salmon, J. Toeppen, L. Van Atta, S. Winters, B. Woods, C. LaFiandra and D. Bruns

This paper was prepared for submittal to

Third Annual International Conference on Solid State Lasers for Application to Inertial Confinement Fusion Monterey Convention Center Monterey, CA

June 7-12, 1998

August 17,1998

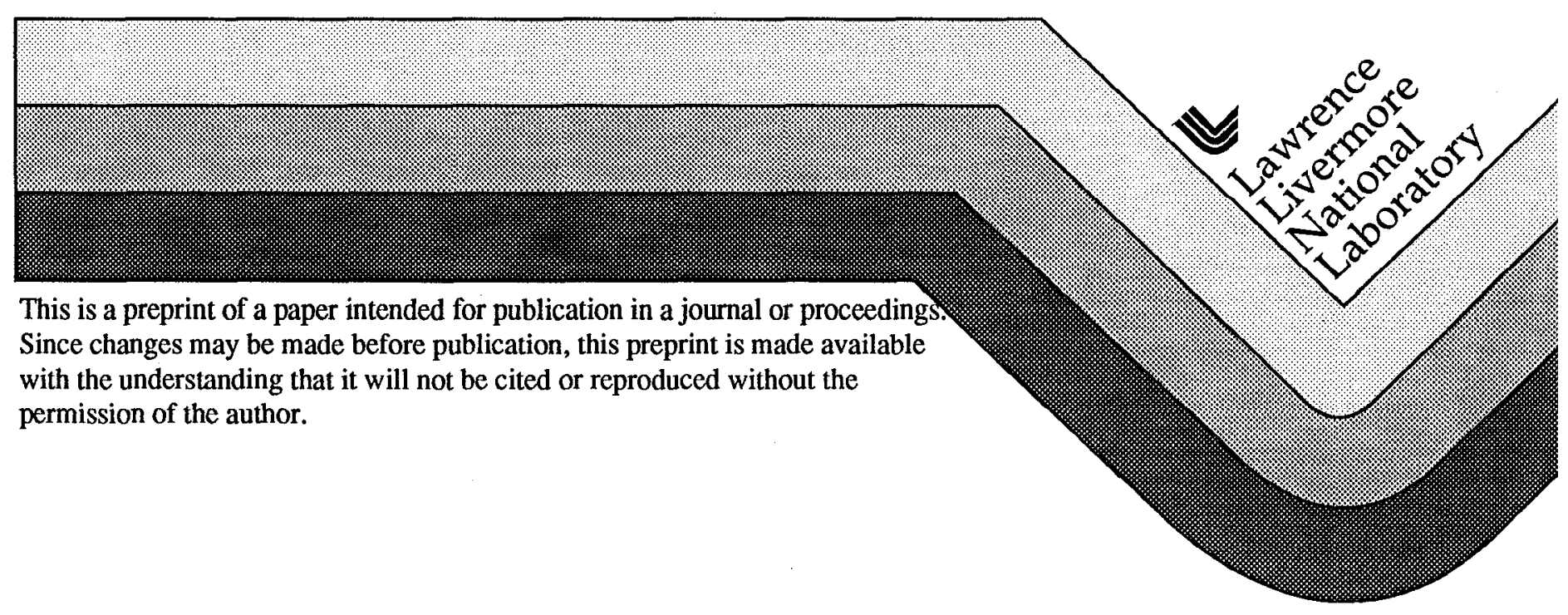




\section{DISCLAIMER}

This document was prepared as an account of work sponsored by an agency of the United States Government. Neither the United States Govemment nor the University of California nor any of their employees, makes any warranty, express or implied, or assumes any legal liability or responsibility for the accuracy, completeness, or usefulness of any information, apparalus, product, or process disclosed, or represents that its use would not infringe privately owned rights. Reference herein to any specific commercial product, process, or service by trade name, trademark, manufacturer, or otherwise, does not necessarily constitute or imply its endorsement, recommendation, or favoring by the United States Government or the University of California. The views and opinions of authors expressed herein do not necessarily state or reflect those of the United States Government or the University of Califomia, and shall not be used for advertising or product endorsement purposes. 


\title{
The National Ignition Facility (NIF) Wavefront Control System
}

Richard Zacharias, Erlan Bliss, Mark Feldman, Andrew Grey, Mark Henesian, Jeff Koch, Janice Lawson, Rick Sacks, Thad Salmon, John Toeppen, Lewis Van Atta, Scott Winters, and Bruce Woods

Lawrence Livermore National Laboratory

700 East Ave., Livermore, CA 94550

Carlo LaFiandra

Raytheon Optical Systems Inc.

100 Wooster Heights Road, Danbury, CT 06810-7589

\author{
Don Bruns \\ ThermoTrex Corporation \\ 10455 Pacific Center Court, San Diego, CA 92121-4339
}

\begin{abstract}
A wavefront control system will be employed on NIF to correct beam aberrations that otherwise would limit the minimum target focal spot size. For most applications, NIF requires a focal spot that is a few times the diffraction limit. Sources of aberrations that must be corrected include prompt pump-induced distortions in the laser slabs, thermal distortions in the laser slabs from previous shots, manufacturing figure errors in the optics, beam off-axis effects, gas density variations, and gravity, mounting, and coatinginduced optic distortions.

The NIF Wavefront Control System consists of five subsystems: 1) a deformable mirror, 2) a wavefront sensor, 3) a computer controller, 4) a wavefront reference system, and 5) a system of fast actuators to allow the wavefront control system to operate to within one second of the laser shot. The system includes the capability for in situ calibrations and operates in closed loop prior to the shot. Shot wavefront data is recorded.
\end{abstract}

This paper describes the function, realization, and performance of each wavefront control subsystem. Subsystem performance will be characterized by computer models and by test results. The focal spot improvement in the NIF laser system effected by the wavefront control system will be characterized through computer models.

\footnotetext{
*Work performed under the auspices of the U.S. Department of Energy by Lawrence Livermore National Laboratory under Contract No. W-7405-Eng-48.
} 


\section{SUMMARY}

With thc baseline wavefront control system, NIF is expected to meet its spot size requirement. The addition of the wavefront control system allows about $50 \%$ more energy to enter the ICF target than would enter without it.

The NIF Wavefront Control System design has evolved from those of wavefront control systems successfully employed on previous LLNL laser systems. The system is designed to minimize cost by sharing beam sampling and relay optics with alignment and diagnostics functions.

To meet the defense-related spot size goal, additional systems would need to be added that are not currently in the baseline design.

\section{OVERVIEW}

A primary requirement for NIF is that each beam shall deliver its design energy into a $600 \mu \mathrm{m}$ hohlraum inertial confinement fusion (ICF) target. The total design energy for 192 beams is 1.8 Megajoules. A defense-related goal for the system is that $50 \%$ of the design energy and power should be contained within a $100 \mu \mathrm{m}$ focal spot at the target plane. This is about twice the diffraction-limited $80 \%$ energy spot size.

In order to meet the spot-size requirement and goal, NIF subsystems are designed to limit wavefront aberrations. Optics have stringent specifications for rms surface gradient, power spectral density and surface roughness. ${ }^{1}$ Stringent specifications are also maintained for optical component mounting. NIF systems are designed to mitigate the effects of temperature and humidity variations and vibrations. ${ }^{2}$ An active alignment system is employed to accurately point the beams into the target. ${ }^{3}$ Even with these efforts to control wavefront aberrations, the spot size requirement and goal could not be met without a wavefront control system.

A block diagram of the NIF main laser optical system is shown in Figure 1, with the NIF Wavefront Control System components highlighted. We first summarize the path of the NIF beam, and then describe the wavefront control functions applied to the NIF beam (or the wavefront control beam surrogate). The NIF preamplifier $1 \omega(1.053 \mu \mathrm{m})$ beam enters the main laser chain near the focus of the transport spatial filter (TSF), directed away from the target. The beam exits the filter as a collimated beam that passes through the boost amplifier heading towards the laser main amplifier cavity. A Pockels cell is set to allow the beam to enter the cavity, where it makes four passes through the main amplifier before the Pockels cell is switched to allow the beam to exit. The beam then exits the cavity, passes through the boost amplifier and the TSF and heads towards the target chamber. The beam is frequency-converted to $3 \omega(351 \mathrm{~nm})$ at the target chamber. 




Wavefront Control System Components

Figure 1. Overview of components in NIF layout

Wavefront control functions are implemented as follows. A cw probe beam is co-aligned with the NIF beam prior to injection into the main laser. The probe beam follows the NIF beam path. A 39-actuator large aperture deformable mirror (DM) operates at the far end of the laser cavity where the beam bounces twice. This two-bounce configuration doubles the effective stroke of the DM. At the TSF output, a tilted sampling surface reflects a small fraction of the beam towards a pick-off mirror near TSF focus that sends the sampled beam through relays to the Output Sensor. Within the Output Sensor, a 77lenslet Hartmann sensor (HS) measures the wavefront. The Hartmann sensor's video output is read by a frame-grabber in the wavefront control computer. The computer calculates the surface displacements to be applied to the deformable mirror to correct the wavefront aberrations in the beam.

There are several required and desired functions that the wavefront system should perform. Required functions are to: 1) Control the $1 \omega$ output wavefront (including pointing) of each beam in the time immediately preceding a laser system shot, 2) Apply compensation for previously-measured pump-induced wavefront distortion, 3) Measure beam output wavefront during a laser system shot, and 4) Control the output wavefront (excluding pointing) during routine system operations between shots. A desired function (not a baseline requirement) is to measure and apply compensation for switchyard and Final Optics Assembly wavefront distortion. This will be necessary to meet the defenserelated spot-size goal described above. 
Wavefront control sub-system design requirements (SSDRs) have been flowed down from the NIF primary requirements, including the spot size requirement. ${ }^{4,5}$ Other factors that influenced the wavefront control system SSDRs included expected system cost and experience with Beamlet, the single-beam NIF prototype laser. ${ }^{6}$ These SSDRs are shown in Table 1. The initial design is expected to meet all design requirements, except initially closed-loop bandwidth. The bandwidth is processor-limited, and it is expected that by the time NIF is implemented, faster processors will be available to meet the requirement without major changes to the software or hardware architecture.

\begin{tabular}{|l|l|l|}
\hline Requirement & Value & $\begin{array}{l}\text { Expected NIF } \\
\text { Performance }\end{array}$ \\
\hline Max residual low spatial frequency angle & $\pm 20 \mu$ radians at $1 \omega$ & $\pm 20 \mu$ radians at $1 \omega$ \\
\hline Maximum open-loop time before a shot & $1 \mathrm{~second}$ & 1 second \\
\hline Minimum closed loop bandwidth & $1 \mathrm{~Hz}$ & $0.5 \mathrm{~Hz}$ (upgrade to $1 \mathrm{~Hz}$ ) \\
\hline Number of actuators & 39 & 39 \\
\hline $\begin{array}{l}\text { Compensation range for simple curvature } \\
\text { (double-pass reflected wavefront) }\end{array}$ & 15 waves at $1 \omega$ & 15 waves at $1 \omega$ \\
\hline Order of aberrations corrected & $\leq 4$ th order & $\leq 4$ th order \\
\hline Measurement accuracy at $1 \omega$ & 0.1 waves & 0.1 waves \\
\hline Lenslet spacing & $\leq 1 / 2$ demagnified \\
& $\begin{array}{l}\leq 1 / 2 \text { demagnified } \\
\text { actuator spacing }\end{array}$ \\
\hline
\end{tabular}

Table 1. NIF Wavefront Control System Requirements

In order for NIF to meet its spot size requirement, all aberrators in the system must be minimized (within affordable limits), and it must be assured that the wavefront control system has the stroke required to correct the worst-case aberrations. To this end, a wavefront budget was established, wherein each aberration contributor was expressed in terms of its first 15 Zernike polynomials (minus the three that describe tilt and piston) and the sum taken. The stroke budget resulting from this analysis is shown in Figure 2. Figure $2 \mathrm{a}$ shows that $59 \%$ of the total wavefront control range is consumed by the main laser, $10 \%$ is used by the optical pulse generator, and the remainder is allowed as margin. Of the main laser contribution shown in Figure 2b, 59\% of the aberration is due to the main amplifier, $14 \%$ is due to the boost amplifier, $13 \%$ is due to the polarizer, and the remainder is due to miscellaneous optics. Note that this budget does not include switchyard and final optics aberrations (that the baseline system cannot control), nor does it include residual error of the deformable mirror. 

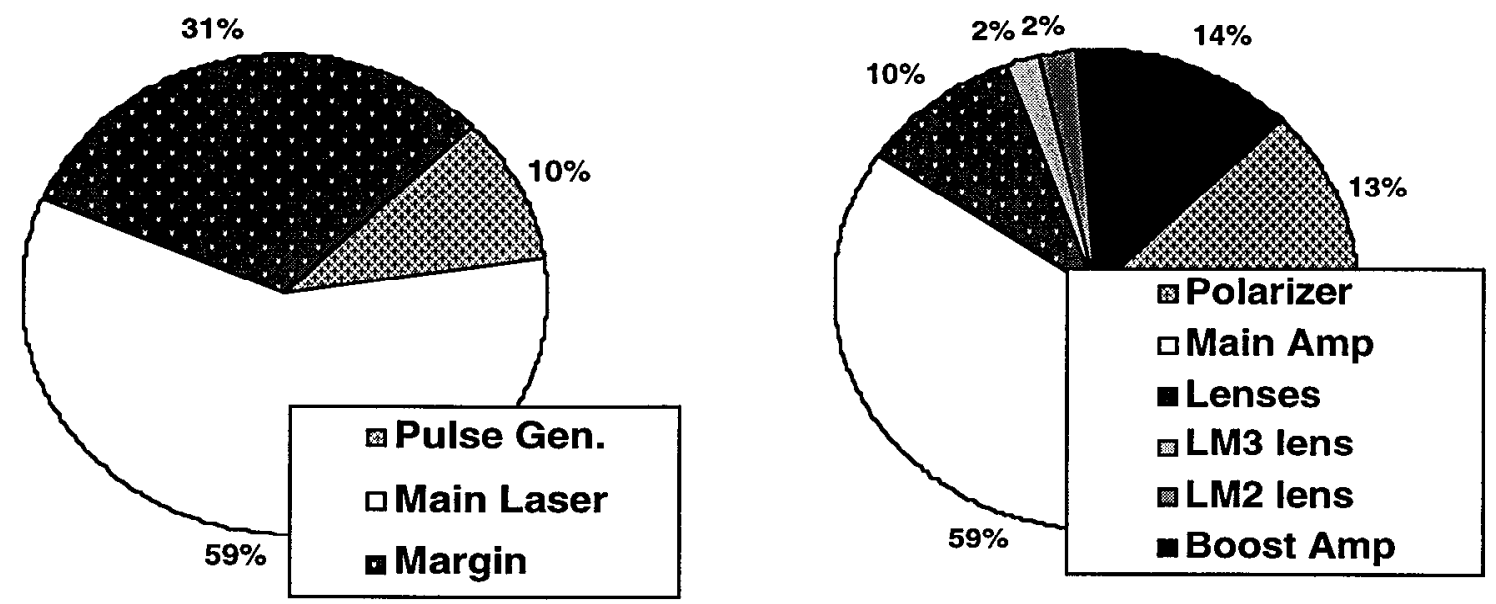

Figure 2. Adaptive optic stroke budget, a) Total budget, b) Main Laser aberrations.

The NIF Wavefront Control System is a new design, but many of the design concepts evolved from LLNL experience with previous laser systems built at LLNL. ${ }^{6,7}$ Portions of the wavefront control system are integrated with NIF alignment and diagnostic functions to reduce cost. For example, the system uses the same laser for the wavefront control probe beam as is used for laser alignment. The wavefront sensor is contained in the NIF diagnostic output sensor so as to avoid the cost of separate beam sampling and relaying optics for the wavefront control function.

The wavefront control system consists of five subsystems for each beam. These are the deformable mirror, the Shack-Hartmann wavefront sensor, the wavefront control computer system, the wavefront reference, and the " $\mathrm{t}_{0}-1$ " system. The " $\mathrm{t}_{0}-1$ " system consists of a set of fast actuators to quickly reconfigure NIF from the wavefront control mode to shot mode. This system is described in a paper by Hartley, et al., and is not described herein. ${ }^{8}$ We now discuss the other subsystems of the NIF Wavefront Control System.

\section{DEFORMABLE MIRROR}

The deformable mirror (DM) must meet stringent performance requirements and must operate in a severe environment. Some of the features and requirements of the DM are shown in Table 2 and some parameters of the environment within which it must operate are shown in Table 3. A particularly stringent requirement is that the DM must have less than 0.025 waves of rms residual error between the DM surface and a true flat surface when the mirror is commanded to be flat in closed loop. The $10 \mathrm{~J} / \mathrm{cm}^{2}$ flashlamp fluence is a particularly severe environmental parameter.

Features and Requirements

39 control points in a hexagonal pattern

Clear aperture of $400 \mathrm{~mm}$ by $400 \mathrm{~mm}$

0.025 waves rms surface residual error (in closed loop to flat) 


\begin{tabular}{|l|}
\hline Replaceable actuators \\
\hline Size and weight compatible with NIF packing density \\
\hline Correction stroke $\geq 4$ waves (surface) \\
\hline Coating: Reflectivity $\geq 99.5 \%, 0.2 \% \leq$ Transmission $\leq 0.5 \%$ \\
\hline Open loop actuator bandwidth $\geq 100 \mathrm{~Hz}$ \\
\hline Actuator linearity $\leq 8 \%$ \\
\hline Actuator lifetime $\geq 10^{9}$ cycles \\
\hline
\end{tabular}

Table 2. Deformable mirror features and requirements.

\begin{tabular}{|l|}
\hline Environment \\
\hline $10 \mathrm{~J} / \mathrm{cm}^{2}$ laser pump flashlamp fluence \\
\hline EMI of 8 gauss and $13 \mathrm{~V} / \mathrm{m}$ in a $200 \mu \mathrm{sec}$ pulse just prior to the laser shot \\
\hline Relative humidity $\leq 3 \%$ \\
\hline Class 50 cleanliness on the optical surface and class 100 for the assembly \\
\hline
\end{tabular}

Table 3. Deformable mirror environmental requirements.

Two companies are currently competing for the deformable mirror contract for NIF, Raytheon Optical Systems Inc., (ROSI), and ThermoTrex Corporation, (TTC). LLNL is also pursuing an in-house design. These three DMs are shown in Figure 3.
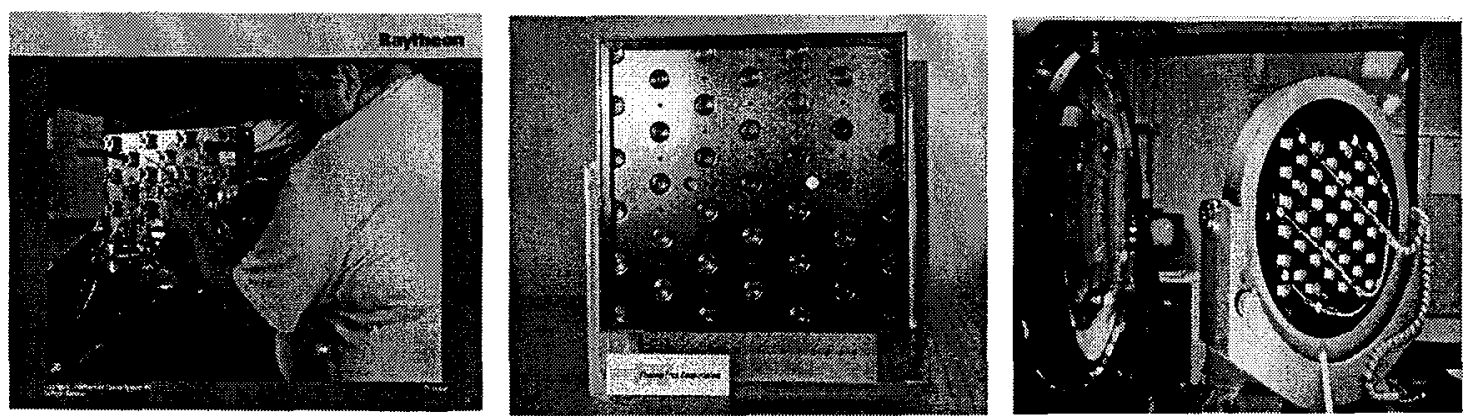

Figure 3. a) ROSI DM, b) TTC DM, c) LLNL DM.

The ROSI mirror employs lead magnesium niobate (PMN) actuators that are bonded indirectly to the mirror faceplate with epoxy. The coupling to the actuator assembly is designed to prevent direct flashlamp light from exposing the epoxy bond and to minimize the scattered light that reaches it. The assembly also allows actuators to be replaced without breaking the bond. ROSI performs the final assembly with the mirror face viewed by a large aperture interferometer so as to minimize pre-assembly distortions that would add to residual manufacturing errors in the assembled mirror. The DM is described in an article written by Carlo LaFiandra. ${ }^{9}$

The ThermoTrex mirror cannot be described at this time, as it uses a proprietary approach. 
As a backup, LLNL is continuing the second-generation development of a deformable mirror that was tested on the Beamlet one-arm NIF prototype laser. This mirror employs PMN actuators and an indirect epoxy bond, as does the ROSI design. The major differences between the two in the actuator force train and the assembly procedure. The actuator is always in compression in the LLNL design but in the ROSI design it can be in either tension or compression. In the LLNL design, the reaction block and faceplate assemblies are lapped flat and then bonded together at each actuator in a butt joint, whereas in the ROSI design, a sliding joint is used to adjust the displacement of each actuator to fit the faceplate until a second epoxy joint sets the actuator as the final assembly step.

Except for the first-generation LLNL mirror, which was tested last year, the three prototypes are undergoing final assembly and have not been tested closed loop. Subassembly tests have been completed for all three designs. For example, we have tested epoxy bond strength in the presence of flashlamp light. We have tested actuators to assure that they survive the EMI of the laser flashlamps during the shot. We have also tested candidate epoxies for mass loss. Since the deformable mirror resides within the same cavity as the NIF main amplifier laser slabs, we must assure that volatile and particulate emissions generated by the mirror do not cause damage to laser slabs or optics coatings when they are exposed to high fluence laser and flashlamp light.

\section{WAVEFRONT SENSOR}

NIF will employ miniature Shack-Hartmann sensors to detect wavefront. ${ }^{10}$ A sketch depicting the operation of the sensor is shown in Figure 4. The sensor has been demonstrated to resolve 0.1 wave at $1.053 \mu \mathrm{m}$. The sensor uses an array of lenslets manufactured by MEMs Optical Systems Inc. Each lenslet generates a focus spot whose position displacement is directly proportional to the local deviation from collimation of the portion of the beam that impinges on it.

In NIF, each Output Sensor is shared by two beams. For most diagnostics, the operator selects which beam is to be viewed, but since the wavefront control system must operate simultaneously for all beams, two beams are spatially multiplexed onto one sensor. ${ }^{11}$

The sensor monitors a beam that has been demagnified to be somewhat smaller than onehalf of the CCD camera array, as shown in Figure $4 b$.

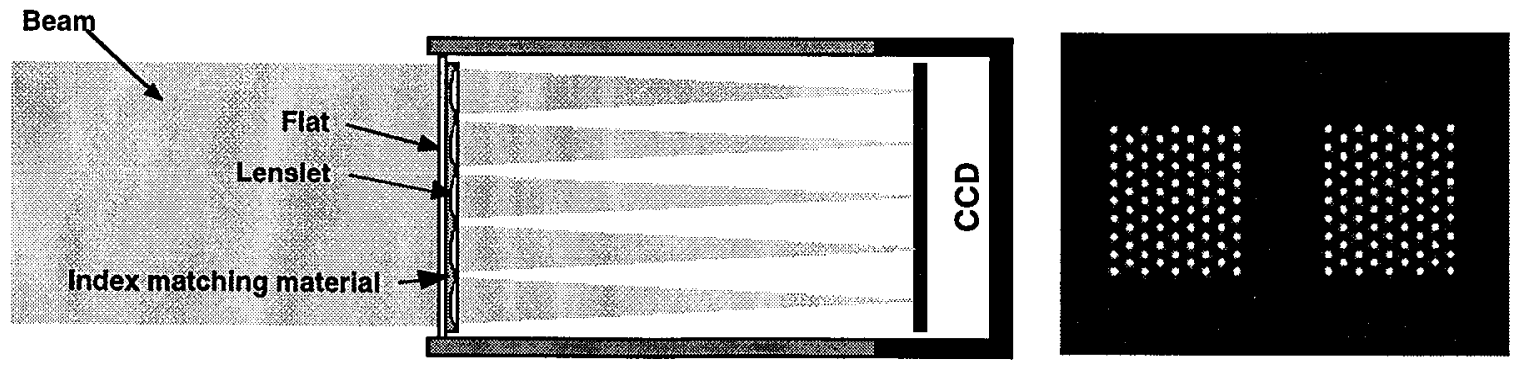

Figure 4. a) Shack-Hartmann sensor sketch. b) NIF sensor CCD spot array image. 


\section{WAVEFRONT REFERENCE}

The wavefront measuring system is calibrated by inserting a wavefront reference fiber at the focal point of the TSF. This concept is shown in Figure 5. Since the fiber light source is smaller than the TSF focal spot, the spot pattern at the Hartmann sensor when viewing the fiber reference beam is the same as the pattern the sensor would see when viewing the probe beam, if the beam and all upstream system components had diffraction-limited performance. The aberrations (imperfections in the separations of the lenslet array focal points) that are seen with the reference inserted are due to aberrations in the measuring system (sampling surface, relay optics, output sensor optics, and the sensor itself). By designing the control system to use the sensor focal spot image of the wavefront reference as the target wavefront to which the system wavefront is controlled, we are forcing the system to generate, as closely as it is able, a perfect focal spot in the TSF. This also implies that aberrations in all the optics beyond the TSF focus, including the TSF output lens, are uncorrected by the baseline wavefront control system.

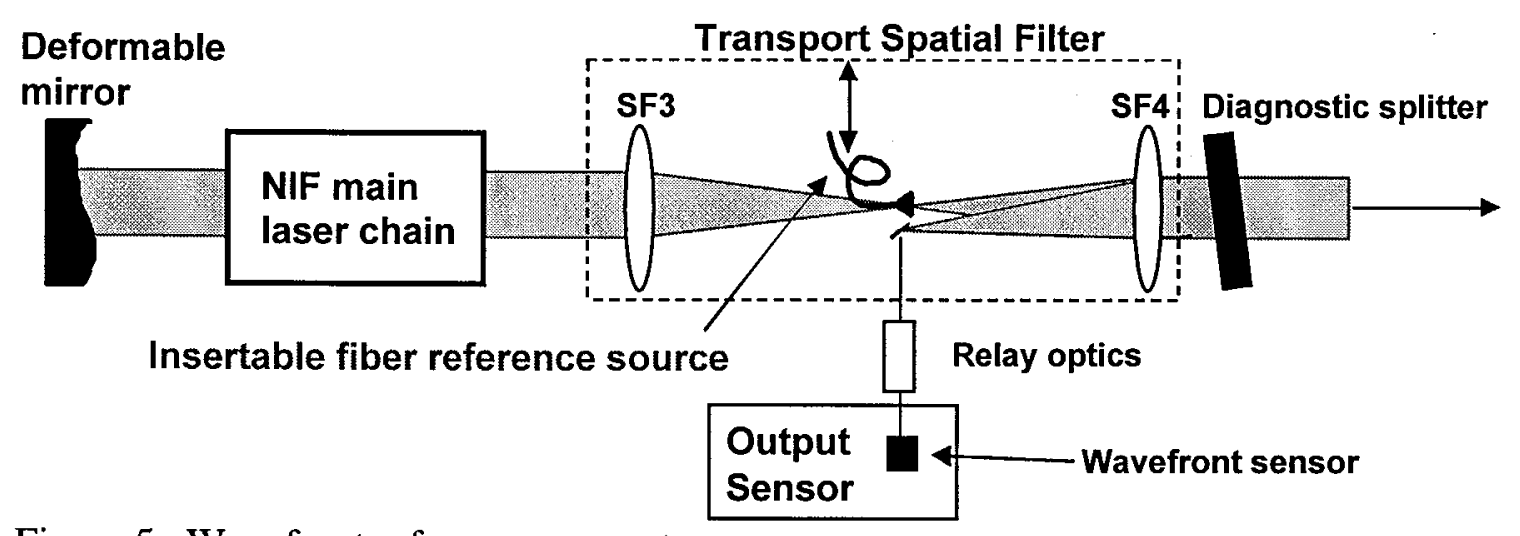

Figure 5. Wavefront reference concept.

\section{WAVEFRONT COMPUTER CONTROL SYSTEM}

A functional block diagram of the NIF wavefront control computer is shown in Figure 6. Initially, the wavefront reference is inserted and its spot positions are measured by the Hartmann sensor creating a reference spot position file. Next, the wavefront reference source is replaced by the probe beam and the wavefront control system is calibrated by an on-line procedure. Each of the 39 actuators is individually poked and pulled relative to the best-flat starting point. The offset for each Hartmann spot is thus related to the displacement of each actuator. From this information, a gain matrix relating actuator movement to Hartmann sensor focal spot movement is derived. ${ }^{7}$

Once the calibrations are complete, the loop is closed wherein the measured Hartmann offsets from the reference positions are multiplied by the gain matrix yielding the actuator offsets to control the mirror to flat (with appropriate loop gain for stability). This is the configuration used during alignment. After alignment is completed and the shot sequence has begun, an additional Hartmann offset file is subtracted from the wavefront sensor data prior to being applied to the gain matrix. These additional offsets 
represent the uncorrected prompt pump-induced wavefront aberrations measured on a previous shot. By subtracting out these offsets, we are setting the wavefront to the conjugate of the expected prompt aberration of the upcoming shot. Thus, at shot time, the wavefront is flat.

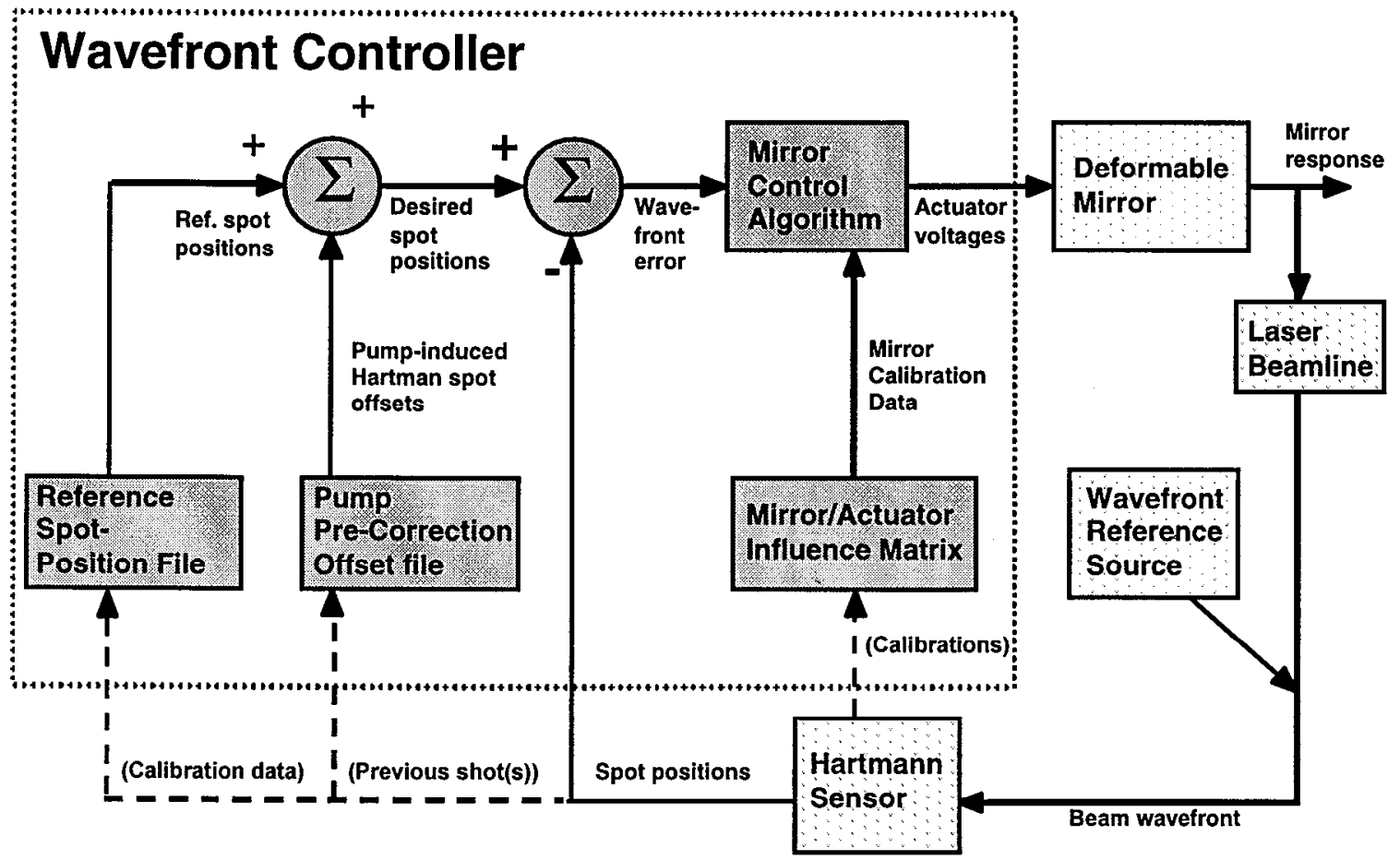

Figure 6. NIF Wavefront Control System block diagram.

The NIF wavefront control computer system uses modular hardware and object oriented software. The NIF facility is expected to be in operation for 30 years, and it is unreasonable to expect to not have to make software or hardware change over that time. By using a modular hardware and software architecture, the system maintainability is improved significantly. The system is designed so that one controller controls one bundle of eight beams.

The control system operates with a closed loop bandwidth of up to $1 \mathrm{~Hz}$. To achieve this, the wavefront sensor is read at a $10 \mathrm{~Hz}$ rate (with a goal of $30 \mathrm{~Hz}$ ). The sensor is readout in standard RS-170 video, which is read by an Active Imaging Snapper 24 frame grabber. The digitized image is fed into a SPARCengine AXI computer that calculates centroids for all 77 lenslet spots and then calculates their offsets from the reference positions. This information is sent via a dedicated ethernet line to the Motorola MVME 2306 controller that calculates the required DM actuator displacements. Each image processor services four Hartmann sensors and eight beams. Each control computer services four DMs. 


\section{WAVEFRONT SYSTEM TEST FACILITY}

The wavefront control system is tested at LLNL in a spccial interferometcr system that can view the deformable mirror surface while the wavefront control loop is closed. A standard practice in the design of a Fizeau interferometer is to minimize the size of the interferometer cavity by keeping the partially-transmitting reference surface adjacent to or as close as possible to the measured surface. This minimizes coherence length degradation, air path density variations, and differential vibrations. Unfortunately, this conflicts with the requirement that the Hartmann sensor see only one surface - the DM to be controlled. This requirement drove our design to the configuration used.

A block diagram of the interferometer system is shown in Figure 7. The YAG beam from a commercial 4' interferometer head (Phase Shift Technologies) returns a portion of its beam at the transmission flat before the beam passes through a splitter and is expanded by a high quality telescope. The expanded beam reflects off of the deformable mirror (or a reference flat) and returns through the telescope. On the return path, the splitter sends a portion of the beam to the Hartmann sensor. Since the Hartmann sensor beam sample is taken within the interferometer cavity, it sees only one reflective surface, thus the loop can be closed. The reference flat replaces the DM for a reference measurement used to subtract out the aberrations in the interferometer path added by the splitter, fold mirrors, and telescope. The entire system resides on a granite slab within a temperature and humidity controlled enclosurc to minimize vibration and air density variation effects which otherwise would be problematic due to the large interferometer cavity.
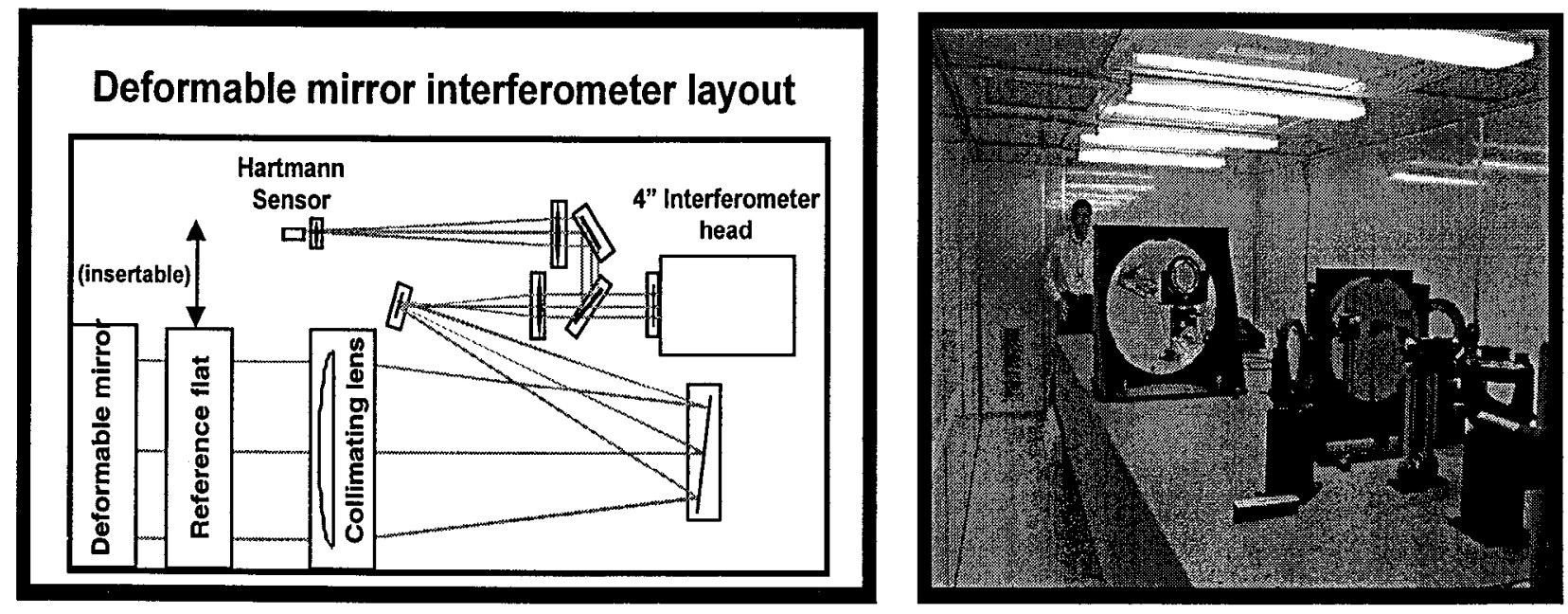

Figure 7. Wavefront Control System Test facility. a) Layout, b) Photograph.

\section{NIF PROPAGATION MODELING OF LASER SPOT SIZE}

We use propagation models to predict system performance. The entire NIF laser has been modeled using the Prop92 laser propagation code. The code models all the beam optics past the initial beam shaper, amplifier and slow thermal aberrations, random large- 
optics aberrations, frequency conversion with non-linear effects, uncorrected gas inhomogeneity, polarizer and mirror coating stresses, and the real-time wavefront correction algorithm, including the Hartmann sensor limitations. This work is described in detail in a paper by Sacks, et al. ${ }^{12}$

The predicted NIF tripled laser focal spot results are shown Figure 8 . The system is first modeled without a wavefront control system. The system is only able to get about $65 \%$ of the total laser power into the ICF hohlraum laser entrance hole. Furthermore, the beam nearfield has high contrast (due to clipping due to poor angular control) and the peak fluence at the final optics has exceeded the maximum "red-line" value. The system modeled to include the wavefront control system focuses almost $95 \%$ of its energy into the target laser entrance hole. The nearfield has much lower contrast, and the peak does not exceed red-line. Thus, the wavefront control system allows over $50 \%$ more laser energy to gainfully (pun intended) enter the target.

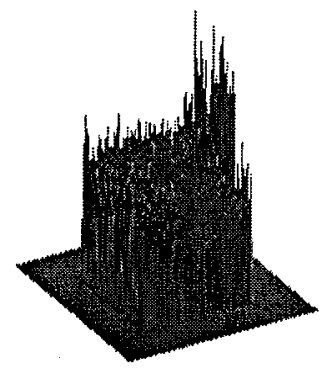

/extern/ras/NIF/bel48.dp000 Jun $209: 59$

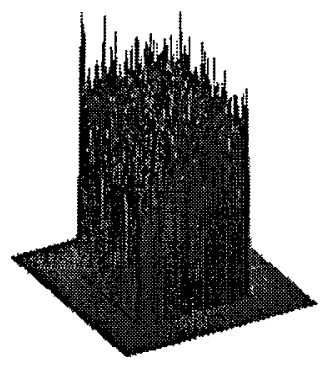

/extern/mos/NIF/bct45.dpoon Moy 29 18:22
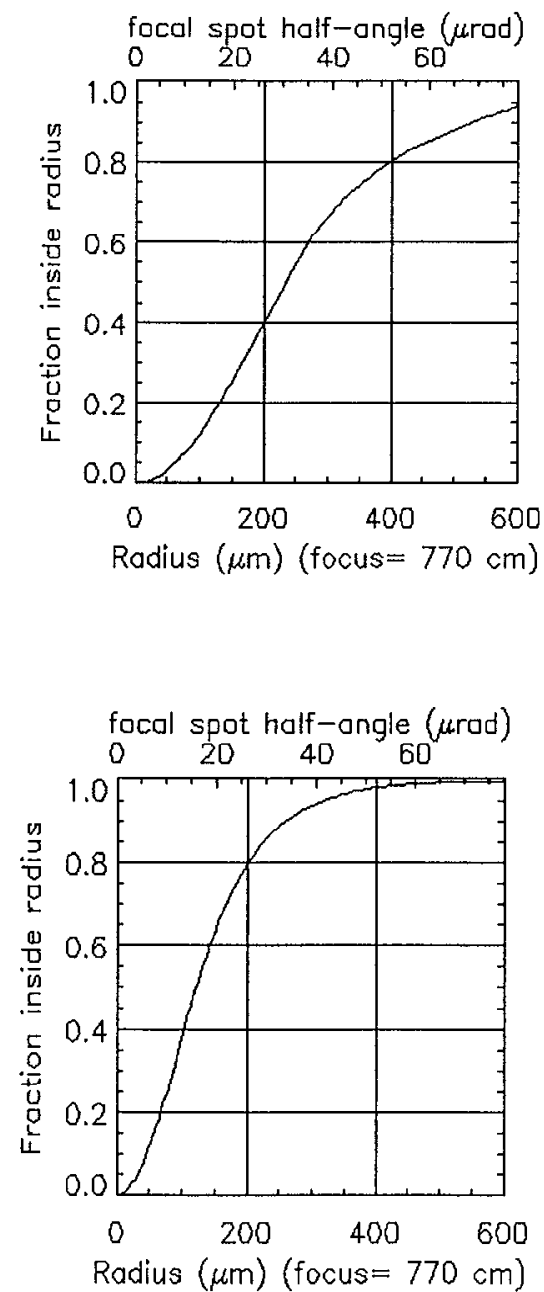

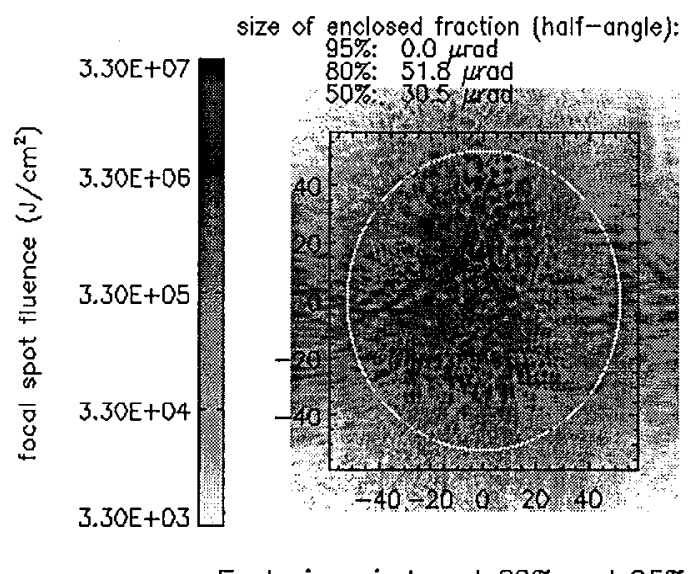

Enclosing circles ot $80 \%$ and $95 \%$

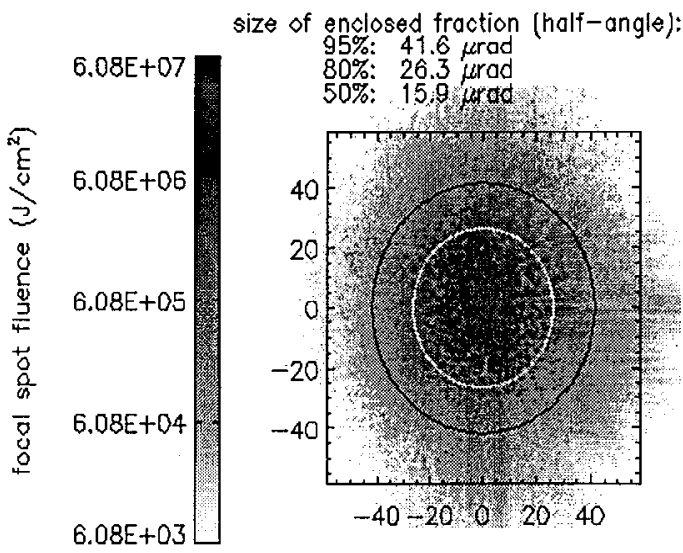

Enclosing circles at $80 \%$ and $95 \%$

Figure 8: NIF output focus spots with (a) and without (b) wavefront control system 


\section{OPTIONAL SUBSYSTEMS FOR IMPROVING SPOT SIZE}

While NIF is expected to meet its spot size requirement, the baseline wavefront control system is not expected to meet the defense related spot-size goal. The primary reasons for this are 1) static aberrations in the optics are expected to have significant energy in spatial frequencies beyond those that can be corrected by 39 actuators over the aperture, and 2) the baseline system does not correct for aberrations in the switchyard and final optics.

There are several options for correcting aberrations in the TSF output lens and those in the switchyard and final optics. The first option is to measure off-line the correction required for each optic and then apply the correction using the Wavefront Control System. The steps are: 1) separately measure the aberrations induced by each optic, 2) determine the total aberration for the suite of optics in cascade, 3) calculate the Hartmann sensor lenslet spot offsets associated with this total aberration, and 4) apply offsets to the target array pattern to pre-compensate. This would require measurements to be made of all switchyard and final optics in their use angles, which would require a significant investment in measurement fixtures.

A second option, depicted in Figure 9, is to place a wavefront sensor on gimbals at target chamber center, and view the wavefront of each beam at that point while the main laser wavefront correction system is operating. The static component of the difference between the wavefront viewed at TCC and the residual aberrations of the baseline wavefront control system would be a measure of the switchyard and final optics static aberrations. Small time-varying gas-density-variation effects could be averaged out. The gimbals would allow one instrument to service all beams. The ability to measure on-line would automatically provide a measurement at optics use angle.

A third option that is being considered, also depicted in Figure 9, would be able to correct switchyard optic aberrations but not final optics aberrations. The advantage of this approach is that it could independently measure the collimation of the beam at the conversion crystals, which would be a significant help during installation. In this correction option, a $1 \omega$ probe laser and wavefront sensor is placed at the precision diagnostic station. A mirror put in place by an existing robot arm inserts the beam from the probe laser into any of the 192 main beam paths directed towards the final optics. For this on-line test, the Integrated Final Optics Module is replaced by a special version containing a reference flat. The wavefront sensor views the round trip aberrations and compares them against those of a round trip when the robot shunts the beam onto a reference flat at the $1 \omega$ laser output position. For this approach, a separate laser and wavefront sensor instrument also must be built, but the existing robot assembly would allow one instrument to service all 192 beams.

None of these options will correct the static high spatial frequency optics aberrations. Since the aberrations are static, a fixed corrector may be used. A paper by Wade Williams presented at this same conference demonstrated that NIF can meet its spot-size 
goal with such a corrector and that the corrector can still do an adequate job with several optics changes. ${ }^{13}$ The near-field modulation created at the injection mirror when the corrector is placed in the small aperture front-end is shown to not cause damage.



Figure 9. Optional non-baseline wavefront control subsystems to improve NIF performance

While none of these optional systcms arc included in the baseline NIF design, they may all be implemented after the system goes on-line, if the need is established.

\section{ACKNOWLEDGMENTS}

This work was performed under the auspices of the U.S. Department of Energy by Lawrence Livermore National Laboratory under contract No. W-7405-Eng-48.

\section{REFERENCES}

1. J.K. Lawson, et al., "NIF Optical Specifications - The Importance of the RMS Gradient Specification", Solid State Lasers for Application to Inertial Confinement Fusion Third Annual International Conference- June 7-12, 1998 Monterey Conference Center, Monterey, California.

2. D.J. Trummer, R.J. Foley, F.S. Shaw, "Stability of Optical Elements in the NIF Target Area Building”, Solid State Lasers for Application to Inertial Confinement Fusion 
Third Annual International Conference- June 7-12, 1998 Monterey Conference Center, Monterey, California.

3. S.C. Sommer and E.S. Bliss, "Beam Position Error Budget Developed for NIF", Solid State Lasers for Application to Inertial Confinement Fusion Third Annual International Conference- June 7-12, 1998 Monterey Conference Center, Monterey, California.

4. W.H. Williams, et al., "NIF's Basic Focal Spot for ICF Shaped Temporal Pulses", Solid State Lasers for Application to Inertial Continement Fusion Third Annual International Conference- June 7-12, 1998 Monterey Conference Center, Monterey, California.

5. W.H. Williams, et al., "NIF's Basic Focal Spot for Flat In Time Pulses", Solid State Lasers for Application to Inertial Confinement Fusion Third Annual International Conference- June 7-12, 1998 Monterey Conference Center, Monterey, California.

6. B.M. Van Wonterghem, et al., "Beamlet Pulse Generation and Wavefront Control System", Inertial Confinement Fusion Quarterly Report, Volume 5, Number 1, Oct.-Dec. 1994.

7. J.T Salmon, et al., "Active and Adaptive Optical Systems" (SPIE - International Society for Optical Engineering, Bellingham, WA, 1991:Proc. SPIE 1452) pp.459-467.

8. R. Hartley, et al., "Wavefront Correction for Static and dynamic aberrations to within 1 second of the system shot in the NIF Beamlet demonstration facility", Solid State Lasers for Application to Inertial Confinement Fusion Second Annual International Conference- October 22-25, 1996 Paris, France.

9. C. LaFiandra, et al., "NIF Deformable Mirror", Solid State Lasers for Application to Inertial Confinement Fusion Third Annual International Conference- June 7-12, 1998 Monterey Conference Center, Monterey, California.

10. J.S. Toeppen, E.S. Bliss, T.W. Long, S.T. Salmon, "A Video Hartmann Wavefront Diagnostic that Incorporates a Monolithic Microlens Array", SPIE International Symposium on Optical Applied Science and Engineering, July 21-26, 1991, San Diego, California.

11. E.S. Bliss, et al., "Design Process for NIF Laser Alignment and Beam Diagnostics", Solid State Lasers for Application to Inertial Confinement Fusion Third Annual International Conference- June 7-12, 1998 Monterey Conference Center, Monterey, California.

12. R. Sacks, et al., "Application of Adaptive Optics for Controlling the NIF Laser Performance and Spot Size", Solid State Lasers for Application to Inertial Confinement 
Fusion Third Annual International Conference- June 7-12, 1998 Monterey Conference Center, Monterey, California.

13. W.H.Williams, "Numerical Simulations of a Phase Corrector Plate for NIF", Solid State Lasers for Application to Inertial Confinement Fusion Third Annual International Conference- June 7-12, 1998 Monterey Conference Center, Monterey, California.

\section{KEYWORD LIST}

Key words used in this paper include: wavefront, adaptive optics, deformable mirror, NIF, lasers, and wavefront sensor. 